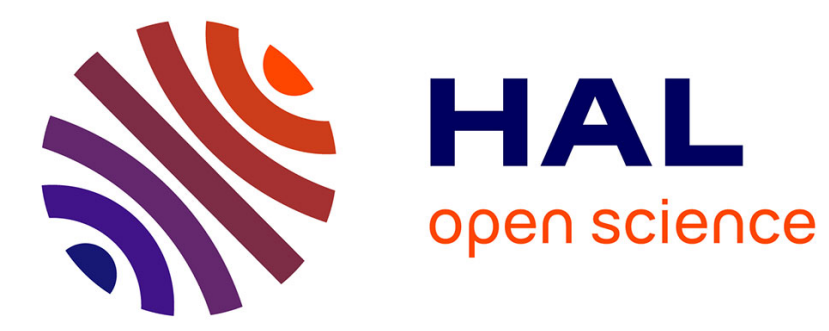

\title{
Dérivation lexicale et variations d'actance: Petits arrangements avec la syntaxe
}

\author{
Alexandre François
}

\section{To cite this version:}

Alexandre François. Dérivation lexicale et variations d'actance: Petits arrangements avec la syntaxe.

Bulletin de la Société de Linguistique de Paris, 2000, 95 (1), pp.15-42. halshs-00135830

\section{HAL Id: halshs-00135830 \\ https://shs.hal.science/halshs-00135830}

Submitted on 9 Mar 2007

HAL is a multi-disciplinary open access archive for the deposit and dissemination of scientific research documents, whether they are published or not. The documents may come from teaching and research institutions in France or abroad, or from public or private research centers.
L'archive ouverte pluridisciplinaire HAL, est destinée au dépôt et à la diffusion de documents scientifiques de niveau recherche, publiés ou non, émanant des établissements d'enseignement et de recherche français ou étrangers, des laboratoires publics ou privés. 


\section{DÉRIVATION LEXICALE ET VARIATIONS D'ACTANCE : PETITS ARRANGEMENTS AVEC LA SYNTAXE}

RÉSUMÉ - La description de langues inconnues suggère à la linguistique générale certains principes méthodologiques, tels que la prise en compte de l'énoncé contextualisé comme unité primordiale de l'observation du sens. Cette méthode rigoureuse prouve sa force en permettant de reconstituer la signification et le fonctionnement, en motlav (Océanie), d'un suffixe fortement polysémique. Rappelant à la fois les questions de préverbation dans les langues européennes, mais aussi les séries verbales ou les morphèmes applicatifs de plusieurs régions du monde, ce suffixe verbal goy nous permet d'explorer, d'une part, le domaine de la sémantique lexicale, et d'autre part, les questions syntaxiques de valence et de transitivité.

Nous définissons ce morphème goy par un "scénario prototypique" impliquant quatre participants A B C D, reliés entre eux par des attitudes d'ordre modal et psychologique. Opérant par métaphore ou métonymie à partir de ces quelques éléments, le motlav dérive un grand nombre de valeurs sémantiques, calculées par combinaison au contexte. Nous montrons à quel prix ce scénario, situé en amont de la phase de formulation, vient ensuite s'insérer dans des structures syntaxiques nécessairement étriquées: il en résulte diverses reconfigurations des relations actancielles.

Dans l'ordre des opérations cognitives présidant à la constitution des énoncés, la syntaxe apparaît alors comme une phase secondaire dans la formulation, consistant à couler l'infini du dicible dans le moule étroit de catégories discrètes et limitées.

Pour qui cherche à décrire pour la première fois une langue sans tradition écrite, le lieu primordial où se manifeste le sens n'est ni le mot ni le morphème, mais l'énoncé contextualisé. La matière première du linguiste est donc la signification globale des énoncés en discours; ensuite, et seulement ensuite, intervient la phase de segmentation et d'analyse syntaxique, au cours de laquelle on tente de distribuer cette signification globale en unités plus petites, internes à l'énoncé. À chaque fois, se pose la double question des limites de cette analyse : jusqu'à quel point peut-on pulvériser le signifié - le sème est-il une unité opératoire, ou seulement le sémème, ce paquet de sèmes associé globalement à un morphème ? Et jusqu'à quel degré peut-on éclater le signifiant : en accordant une valeur linguistique non seulement aux morphèmes euxmêmes, mais à leurs relations réciproques, à leur catégorie syntaxique, à leurs diverses appartenances sémantiques ${ }^{1}$ ?

\footnotetext{
${ }^{1}$ Ces questions s'inspirent notamment de la réflexion d'Alain Lemaréchal $(1989 ; 1997)$ autour de la superposition des marques, et de la sémantique de la syntaxe.
} 


\section{Le morphème goy en motlav : lexique ou syntaxe ?}

Le motlav, langue océanienne (austronésienne) parlée au nord du Vanuatu par env. 1500 locuteurs $^{2}$, présente de nombreux énoncés dans lesquels le verbe ou groupe verbal se termine par une syllabe [yoj], transcrite /goy/, sans qu'il soit facile de lui attribuer une valeur sémantique stable. La première question est de savoir si cette syllabe est un morphème pourvu d'une signification unique, ou si l'on a plusieurs suffixes homonymes, dotés chacun d'un sens différent; au pire, si cette tentative de motivation sémantique échoue, la récurrence de cette syllabe sera imputée au hasard.

Il arrive, tout d'abord, que le test de commutation échoue, au sens où goy ne peut pas être supprimé du lexème verbal : galingoy "comploter", sisgoy "tomber", vatlēggoy "faire des fiançailles", tabeggoy "fermer", comportent obligatoirement cette syllabe finale /goy/, sans qu'il soit possible de rencontrer, ailleurs dans la langue, les mots *vatlēg, *galin, etc. Il est donc exclu de chercher à analyser ces verbes, du moins en synchronie - même si, au bout du compte, on y retrouvera le sémantisme de notre morphème.

Cependant, la grande majorité des occurrences de goy - plus de 215 dans notre corpus spontané - laisse reconnaître un premier élément verbal V, par ailleurs autonome dans la langue : tiggoy "empêcher" [VT] rappelle tig "être debout" [VI] ; vapgoy "bénir" [VT] $\rightarrow$ vap "dire" [VT] ; etgoy "faire attention" [VI] $\rightarrow$ et "voir" [VT], etc. Du point de vue morphologique, tout se passe comme si l'on avait un (ou plusieurs) suffixe(s) goy, isolable(s) par commutation, et compatible(s) avec certains verbes pour en former d'autres. Si le procédé est entièrement productif, i.e. si goy peut se combiner à n'importe quel verbe au gré du locuteur, alors on y verra un morphème autonome, entrant avec le verbe $\mathrm{V}$, à chaque fois, dans une relation syntaxique; en revanche, s'il apparaît que seuls certains verbes sont compatibles avec goy, et/ou que la signification de l'ensemble /V+goy/ ne peut pas être calculée à partir de leurs sens respectifs, alors on parlera davantage d'un suffixe morphologique de dérivation lexicale.

De fait, il apparaît que les deux interprétations sont possibles, ou plutôt qu'elles coexistent dans la langue : dans certaines de ses acceptions sémantiques qu'on verra plus loin (ex. Réactif; Détrimental), goy est productif, et peut virtuellement s'associer à n'importe quel verbe ; dans d'autres (Secret ; "danser sur"), goy ne concerne qu'une poignée de verbes, lesquels sont appris par cœur avec cette valeur.

Pour rendre la chose plus claire et élargir l'angle de vues, il semble déjà possible d'établir des comparaisons avec d'autres langues. Le comportement de goy ressemble assez à celui des préverbes des langues indo-européennes, ou des postverbes de l'anglais. Ainsi, les problèmes sémantiques - et syntaxiques, on le verra - que pose ce morphème du motlav sont du même ordre que ceux qu'implique, par ex., le postverbe anglais $u p$ : peut-on trouver un sens récurrent pour up dans les groupes verbaux climb up "grimper (vers le haut)", catch up "rattraper", pop up "surgir", give up "se désister", sit up "être assis droit", shut up "se taire" ?

Ici, comme pour le motlav, il faut accepter une réponse contradictoire : d'un côté, up apparaît souvent dans des syntagmes colexicalisés et sémantiquement opacifiés, appris tels quels par les locuteurs ; de l'autre côté, certaines valeurs de ce même (?) up sont suffisamment récurrentes (ex. "vers le haut" ; "instantanément") pour permettre

\footnotetext{
${ }^{2}$ Nous avons présenté en détails la morpho-phonologie de cette langue dans François (1999).
} 
aux locuteurs eux-mêmes de forger / interpréter de nouveaux syntagmes, par simple analogie. L'étude des langues ne cesse de révéler ${ }^{3}$ l'importance fondamentale, pour modéliser les comportements langagiers, de cette dualité entre formes opaques, apprises telles quelles par des locuteurs passifs, et transmises par "inertie" - et formes (re)motivées, associées, en amont ${ }^{\mathrm{et}} / \mathrm{ou}$ en aval, à des règles plus ou moins productives, de la part de sujets cognitivement actifs. Cette ambivalence objets appris / objets construits recouvre en partie l'opposition classique ${ }^{4}$ entre lexique et syntaxe ; sauf que loin de s'opposer, ces deux fonctionnements langagiers apparaissent plutôt comme deux aspects, resp. synthétique $v s$. analytique, d'un seul et même mécanisme : la signification.

\section{Problèmes sémantiques}

\subsection{Un casse-tête lexicologique}

Mais pour savoir que certaines valeurs de goy sont opaques en synchronie, il faut déjà avoir cherché à dégager celles qui lui sont associées le plus régulièrement: celles-ci formeront la signification nucléaire de ce morphème, la seule qui soit productive. Ainsi, pour l'anglais up, tout le monde s'accorderait à donner comme centrale la valeur "vers le haut", car elle est à la fois la plus fréquente et la plus transparente.

En réalité, contrairement à la plupart des mots du motlav, goy oppose à l'analyse une incroyable résistance sémantique : les valeurs qu'on peut lui attribuer sont à la fois très nombreuses et difficiles à réduire à l'unité, comme le suggère le tableau suivant. Nous y présentons, d'un côté, le sens et la valence du verbe $\mathrm{V}$, avant d'esquisser brièvement ceux que prennent les combinaisons de ces verbes ${ }^{5}$ avec le suffixe goy.

\begin{tabular}{|c|c|c|c|c|}
\hline \multicolumn{3}{|c|}{ VERBE V } & \multicolumn{2}{|l|}{ VALEURS DE V+ goy } \\
\hline tēy & tenir & VT & retenir $;(\mathrm{se})$ couvrir (le visage...) & VT \\
\hline vap & dire & VT & interdire à ; bénir & VT \\
\hline hag & être assis & VI & squatter (un endroit) ; assister (qqn) & VT \\
\hline hoghog & offrir & VT & se fiancer à, se réserver (une femme) & VT \\
\hline yon̄teg & entendre & VT & écouter attentivement & VT \\
\hline balbal & couper & VT & couper $($ cheveux...) régulièrement & VT \\
\hline tig & debout & VI & se relever [VI] ; protéger; empêcher & [VT] \\
\hline hey & se vêtir de & VT & revêtir (sa tête...) d'un habit & VT \\
\hline mitiy & dormir & VI & se reposer de ; surveiller en dormant & VT \\
\hline et & voir & VT & faire attention à ; s'occuper de & VT \\
\hline lak & danser & VI & danser sur (ипе тиsique) & VT \\
\hline van & aller & VI & aller chercher $[\mathrm{VT}] ;($ temps $)$ s'écouler & [VI] \\
\hline
\end{tabular}

\footnotetext{
${ }^{3}$ Nous avions déjà relevé ce point dans un autre domaine linguistique, celui de la phonologie / morpho-phonologie (François 1999: 458).

${ }^{4}$ Andrew Pawley $(1996 ; 1993)$ mène une réflexion poussée sur les limites de ces deux catégories ; les exemples qu'il cite sont assez proches des nôtres (séries verbales en Kalam, langue papoue non-austronésienne).

5 Par manque de place, nous présentons les verbes isolés plutôt que des énoncés complets ; ces derniers seront donnés en annexe, dans l'entrée goy du dictionnaire.
} 
Ces quelques exemples suggèrent la difficulté d'évaluer précisément l'apport sémantique de goy. Ce morphème reçoit tantôt des valeurs bénéfactives - "bénir", "protéger", "s'occuper de", "assister" -, tantôt des valeurs détrimentales - "empêcher", "interdire", "retenir", ce qui est déjà paradoxal. D'autres emplois ne se laissent pas classer facilement dans l'une ou l'autre de ces deux valeurs: "danser sur (une musique)" n'est ni bénéfactif ni détrimental, et l'on serait tenté de définir goy, faute de mieux, comme un vague "relateur syntaxique", correspondant aussi bien à nos prépositions sur, de, à, qu'à certains préverbes (re- dans retenir, revêtir, etc.); sans parler de significations qui ne sont guère "relationnelles", comme attentivement ou régulièrement... L'inconvénient d'une telle solution générale, si élégante soit-elle, est précisément d'être trop puissante : car même si la glose "relateur" réussit à englober tous les cas où goy apparaît, elle ne permet pas de comprendre ceux où goy est exclu. Surtout, elle ne fournit pas une modélisation satisfaisante des opérations sémantiques qui sont associées à ce mot, en termes de conditions nécessaires et suffisantes d'apparition dans le discours ${ }^{6}$.

Il apparaît beaucoup plus fécond de supposer une signification première (très) précise, de laquelle les diverses acceptions de goy seraient dérivées, par figure stylistique (métaphore, métonymie) ou autre. Une première phase de l'exploration consiste à réunir ensemble les effets de sens qu'on peut considérer comme similaires : se constituent alors des familles d'énoncés ${ }^{7}$, caractérisées par une certaine cohérence interne - indépendamment des rapports existant entre ces familles. Voici un aperçu de cette première classification :

- valeur ALLER CHERCHER : Na-trak so ni-van goy na-mtewot! Il faut une voiture pour aller chercher un blessé ! • Kēy tềvēy goy nō-mōmō. Ils plongent chercher du poisson (ils font de la pêche sous-marine). $・$ Nok so at goy na-plastik. Je me penche pour (prendre) le sac plastique.

- valeur EMPÊCHER: Roland kē ni-têytēy goy kōyō. Roland les a empêchés (de se battre). • Nē-dēmdēm mino, ni-vit ni-akak goy. Ma pensée est obnubilée par les étoiles. Mahē tō-qō̄n goy qiyig dōyō ! [la nuit va tomber sur nous] L'obscurité va nous empêcher de continuer!

- valeur REFERMER : qul goy no-totgal recoller une photo - gon goy na-mteēm verrouiller la porte ...

Nous avons constitué pas moins d'une vingtaine de ces "familles d'énoncés", correspondant à autant (?) d'acceptions possibles pour goy. Ces 20 tiroirs sémantiques seront présentés en annexe, avec, à chaque fois, plusieurs énoncés représentatifs.

Dans un deuxième temps, nous avons cherché à ordonner ces acceptions entre elles, afin de retracer un possible cheminement sémantique menant des unes aux autres, et dégager une éventuelle cohérence sémantique globale. Malgré le grand éventail de significations liées à goy, il nous est apparu possible de proposer une

\footnotetext{
${ }^{6}$ C'est aussi là, sans doute, le reproche que l'on peut adresser à la façon dont J.-J. Franckel cherche à redéfinir abstraitement le préfixe re- ou le nom lit, en faisant appel à un nombre minimal d'opérations (Franckel \& Lebaud 1992).

${ }^{7}$ L'unité pertinente pour le linguiste, comme nous l'avons suggéré dans l'introduction, n'est pas le mot ni le syntagme, qui sont inclassables - ex. tig goy présente de nombreuses valeurs distinctes. C'est l'énoncé en contexte, seule unité qui soit dotée d'une signification stable pour l'observateur : dans tel énoncé précis, goy aura un effet de sens (éventuellement complexe) et un seul. Cf. Gross (1999: 117) : "postuler la phrase comme unité de base".
} 
hypothèse unificatrice, que nous présentons ci-dessous. Outre qu'elle rend assez bien compte de la plupart des 215 occurrences de notre corpus, cette hypothèse est confirmée par notre intuition de locuteur du motlav - intuition certes imparfaite, mais suffisante pour forger de nouveaux syntagmes en goy, et interpréter sans trop d'erreur des combinaisons inédites.

Nous ne pouvons pas entrer ici dans les détails du raisonnement sémantique. Nous mentionnerons simplement un point concernant l'orientation vers les participants au procès, en vue de mieux aborder les questions syntaxiques qui suivront.

\subsection{Un schéma général à quatre participants}

Dans un premier temps, nous partirons des acceptions (ou "familles d'énoncés") dans lesquelles l'apport sémique de goy est le plus net. Ainsi, si l'on examine l'ex. (1'),

(1) Nēk ma-hag.

tu PFT-assis

Tu es assis.

(1') Nēk ma-hag goy ni-vidio den no !

tu PFT-assis goy la-télé ABL moi

Assis comme tu l'es, tu m'empêches de voir la télé!

on constate que goy modifie le comportement du verbe hag "être assis" de deux façons :

- Apport sémantique: goy indique la conséquence de la première action "être assis", et particulièrement les effets sur autrui : en se trouvant dans telle position, le sujet (nēk) produit un certain effet sur qqn d'autre (no) - il le gêne.

- Apport syntaxique: goy change la valence du verbe intransitif hag, pour constituer un verbe complexe hag goy, qu'on peut décrire comme transitif direct : "être assis devant (qqch) / occulter (qqch) en étant assis". Tout se passe comme si goy, au moins dans cet exemple, marquait une diathèse progressive, à effet transitivant

L'inconvénient d'une approche purement syntaxique de l'énoncé (1'), en termes de transitivité, est qu'elle n'entérine que l'ajout d'un actant, à savoir l'objet (ni-vidio) du verbe hag goy. Quant à la seconde addition, à savoir le syntagme prépositionnel den no "de moi", elle n'est pas censée entrer dans la valence de ce verbe : si l'on définit strictement la valence par le nombre d'actants directement régis par le verbe, on est simplement passé d'un verbe monovalent en (1), à un verbe divalent en (1').

Pourtant, l'intuition perçoit bien que le "circonstant" den no est également un apport de goy, d'autant plus qu'il est impossible en (1); même si c'est den qui semble introduire cet élément, sa signification dans l'énoncé se calcule, avant tout, à partir du suffixe verbal ${ }^{8}$. Par conséquent, on est tenté de considérer que le suffixe goy ne met pas en jeu simplement deux participants ("toi" et "la télé"), mais bel et bien trois ("toi", "la télé", et "moi"). Simplement, ces trois actants ne peuvent pas être associés directement au verbe, comme nous le verrons plus loin dans l'analyse syntaxique [§3.4].

\footnotetext{
${ }^{8}$ De la même façon, il est admis que dans une phrase française comme J'ai donné le livre à ma sœur, le rôle sémantique qui sera attribué à "ma sœur" (Bénéficiaire, et plus précisément Donataire) résulte non seulement de la préposition à, mais aussi, c'est important, de la valence / du sémantisme de donner.
} 
L'énoncé (2') rappelle le type de relations sémantiques rencontrées en (1'), notamment entre le sujet et l'objet du verbe :

(2) Na-naw na-hal.

la-mer STATIF-flotter

La mer "flotte" (i.e. ne coule pas comme une rivière)

(2') Iñglan, na-naw na-hal goy.

Angleterre, la-mer STATIF-flotter goy

L'Angleterre est une île. [lit. la mer flotte + fait obstacle]

On peut comparer terme à terme (2') et (1') : la mer est à l'Angleterre ce que "toi" était à la télévision, c'est-à-dire un obstacle. D'autre part, même s'il n'est pas syntaxiquement exprimé en (2'), on voit bien que goy a également introduit, du fait de son sémantisme même, un participant invisible: car présenter un $\mathrm{X}$ comme un "obstacle", implique nécessairement la référence à un sujet Y, celui-là même qui se trouve gêné par l'obstacle en question. En (1'), le sujet ainsi "empêché" était no ("moi"); en (2'), c'est implicitement "les gens / on", tous ceux qui voudraient atteindre l'Angleterre en partant du continent.

Comme nous allons rencontrer souvent ces mêmes relations sémantiques entre participants, indiquons déjà les symboles que nous avons choisis pour les désigner. Parmi les lettres arbitraires A B C D, qu'on verra plus loin, nous avons déjà rencontré :

- A : sujet du verbe $\mathrm{V}$ et du complexe verbal V+goy ;

- B : chose qui se trouve cachée / recouverte / protégée par l'action $\mathrm{V}+\mathbf{g o y}$;

- D : chose ou personne qui se trouve empêchée / gênée par V+goy.

Dans les deux exemples que nous venons de détailler, on observe des relations parallèles entre les participants : un agent ${ }^{9}$ A ("toi" / "mer"), de façon volontaire ou non, empêche une personne D ("moi" / quiconque) d'accéder à un objet B ("télé" / "Angleterre"). Dans les deux énoncés, l'objet syntaxique du verbe $\mathrm{V}+$ goy est $\mathrm{B}^{10}$, tandis que l'élément $\mathrm{D}$ se trouve soit périphérisé sous forme de circonstant (1'), soit complètement implicite (2').

Avant d'aller plus loin, faisons entrer un quatrième participant dans la bataille. Comparons à nouveau les verbes $\mathrm{V} / \mathrm{V}+\mathbf{g o y}$ :

(3) Hey na-hat anen !

porter le-chapeau là

Mets donc ce chapeau!

(3') Hey goy ni-qti mi na-hat anen !

porter goy ta:tête avec le-chapeau là

Protège-toi donc la tête avec ce chapeau!

Ce couple d'énoncés appelle nombre de commentaires, que nous verrons plus loin [§3.4]. Pour l'instant, contentons-nous de relever la pertinence sémantique d'un nouveau participant $\mathbf{C}$, dans les énoncés en goy. En effet, s'il est vrai que "ta tête"

\footnotetext{
${ }^{9}$ Le terme d'agent est à prendre ici au sens large, sans préjuger de la valeur agentive ou non du procès. Le motlav est une langue SVO à système "accusatif" / non-ergatif [cf. § 3.1].

${ }^{10}$ Dans l'énoncé (2'), cet objet est thématisé, puis repris par anaphore zéro sur le verbe ; cela est normal, en motlav, pour les objets inanimés. Cf. français Le cinéma, j'adore !
} 
correspond bien à la définition sémantique (et à la description syntaxique comme COD) de l'élément $\mathrm{B}$, en revanche le "chapeau" dont il est ici question n'a rien à voir avec celle de D : ce chapeau n'est pas le "gêné", mais le "gêneur", car il est utilisé pour protéger sa tête d'un danger extérieur (ex. le soleil, D). D'ailleurs, alors que D était marqué au moyen de la préposition ablative den en (1'), C est ici précédé de la préposition instrumentale mi, signalant la différence sémantique entre ces deux participants $\mathrm{C}$ et $\mathrm{D}$.

À strictement parler, on ne peut pas dire que C ("le chapeau"), dans l'énoncé (3'), résulte de l'adjonction du suffixe goy, puisqu'il était déjà présent en (3), comme objet de hey. Néanmoins, il est clair que cet élément $\mathrm{C}$ entre de plein droit dans la configuration sémantique associée au syntagme verbal V+goy. On peut l'ajouter aux trois éléments $\mathrm{A}, \mathrm{B}, \mathrm{D}$ déjà définis :

- C : moyen par lequel A protège B contre D : instrument de l'action $\mathrm{V}+\mathbf{g o y}$.

Pour abréger, on pourra gloser nos quatre éléments ainsi : A le protecteur; B le protégé ; C la protection ; D l'agresseur.

\subsection{Scénario prototypique et dérivation sémantique}

Ces quatre gloses métaphoriques permettent de définir, à partir des trois exemples précédents, un scénario prototypique ${ }^{11}$ associé au suffixe verbal goy, et mettant effectivement en jeu, au maximum, quatre participants :

SCÉNARIO TYPIQUE de goy: Un sujet A recouvre un objet B au moyen d'un objet couvrant $\mathrm{C}$, spéc. de telle sorte qu'il empêche, volontairement ou non, une tierce personne D d'accéder à B. D'où $\mathbf{A}$ interpose $\mathbf{C}$ pour protéger $\mathbf{B}$ et $/$ ou pour repousser $\mathbf{D}$.

Il arrive, quoique rarement, que les quatre éléments A B C D soient tous explicités dans un même énoncé :

(4) Hey goy na-ȳ̄ē den ne-nem mi na-trausis. porter goy tes:jambes ABL moustique avec pantalon

Protège-toi les jambes des moustiques avec un pantalon.

Mais le plus souvent, le scénario prototypique associé à goy ne se retrouve représenté que partiellement dans les énoncés, ou de façon métaphorique. Ainsi, en (1'), l'instrument couvrant $\mathrm{C}$ se confondait avec l'agent $\mathrm{A}$, car celui-ci cachait $\mathrm{B}$ avec son propre corps. Ce sous-schéma se retrouvera souvent lorsque $\mathrm{V}$ est un verbe de position corporelle, sémantiquement statif : hag goy "empêcher l'accès en étant assis", tig goy "...en étant debout", en goy "...en étant allongé", etc.

D'autre part, les valeurs psychologiques éventuellement associées aux gloses "A protège $\mathrm{B}$ pour repousser $\mathrm{D}$ ", doivent être comprises comme renvoyant à une configuration prototypique, selon laquelle le protecteur A est plutôt <HUMAIN ACTIF CONSCIENT FORT>, et protège un objet B plutôt 〈INANIMÉ INACTIF FAIBLE〉, au moyen d'un objet $\mathrm{C}$ plutôt <INANIMÉ FORT>, contre un élément D typiquement <HUMAIN ACTIF FORT AGRESSIF>.

${ }^{11}$ Cf. le prototype chez Kleiber (1990), le sémème revisité par Touratier (1996) et les radial cognitive structures de Lakoff (1987). 
Nous verrons [§ 4.2] l'utilité de considérer comme centrales ces valeurs prototypiques, car c'est à partir d'elles que se constitueront divers effets de sens liés à goy. Cependant, il convient de souligner dès maintenant que chacune de ces catégories peut être "déformée" de façon métaphorique : ex. en (2'), la mer $(A=C)$ n'est pas animée, et n'agit pas de façon consciente / agentive ; et doit-on vraiment considérer que cette mer "protège" l'Angleterre (B) de terribles agresseurs (D) venus du continent? Nous laisserons ici cette question en suspens.

Le détail des différentes acceptions de goy apparaît dans l'entrée du (futur) dictionnaire du motlav, reproduite ici en annexe. Les différentes acceptions de ce mot sont ordonnées, de façon à modéliser un cheminement sémantique vraisemblable, à partir du scénario prototypique que nous venons de reconstituer. Bien sûr, on se gardera bien de confondre ce dernier, sorte de dénominateur commun des emplois attestés en synchronie, avec un éventuel sens étymologique : celui-ci pourrait être très différent, tant il est vrai que les extensions et réanalyses sémantiques que connaissent les mots, au cours du temps, peuvent transformer leur configuration sémique ${ }^{12}$. D'ailleurs, l'étymologie de goy est connue : son étymon en POc (Proto-Océanien) est une racine *koro "village fortifié", donc sans doute un nom. Le développement de significations abstraites, et l'emploi comme second élément du syntagme verbal, serait propre aux (94) langues du Nord-Centre Vanuatu ${ }^{13}$; en outre, il semble bien que le motlav soit une des langues où cette extension sémantique ait été la plus radicale.

\subsection{L'implication des participants : continu ou discret ?}

Pour chaque type d'emploi de goy, nous faisons précéder la définition par un schéma simple reprenant les quatre lettres A B C D. En réalité, chaque acception de ce suffixe est corrélée à une certaine configuration de ces quatre éléments, lesquels peuvent être présents, absents, ou encore présents d'une manière implicite (parenthèses) ou métaphorique (guillemets simples). Par exemple, le sens $\mathrm{n}^{\circ} 7$ "s'approprier, rendre sacré, bénir" est estampillé du label $\left[A, B,{ }^{\prime} C^{\prime},(D)\right]$. Ce dernier signifie que dans cet emploi de goy, A et B sont normalement présents - i.e. soit explicités dans l'énoncé, soit implicités de telle sorte que l'auditeur reçoive l'instruction de les reconstituer. En revanche, l'instrument $\mathrm{C}$, à savoir le moyen par lequel A s'approprie / sacralise $\mathrm{B}$, a une valeur suffisamment métaphorique pour être simplement suggérée par le verbe, et rarement explicitée ; d'autre part, l'agresseur D est, certes, reconstituable, mais il n'a que très rarement de saillance discursive (pragmatic saliency) dans le discours. Ainsi, pour l'action de "bénir", les deux participants obligatoires sont A le prêtre chrétien - et B - l'endroit hanté / la personne possédée / la nourriture ; on ne précise jamais C (les prières...), ni D (les esprits païens, la présence du Diable...), lesquels n'ont qu'une semi-présence du point de vue thématique.

Parallèlement, tous les termes pour "se fiancer" emploient goy : akgoy, vatlēggoy, hoghoggoy. Il s'agit toujours, pour un jeune homme A, de se réserver / s'approprier une jeune fille $B$, en empêchant d'autres hommes (D) d'y accéder ; entre B et D, A interpose un mur symbolique $\mathrm{C}$, à savoir des cadeaux de fiançailles - monnaie de

\footnotetext{
12 Robert Blust propose ici le concept de "fragmentation sémantique" (1987), en particulier à propos de la racine POc *koro (comm. pers.).

${ }^{13}$ Cf. Pawley (1976), Clark (1985: 210) : outre un nom "enclos" bien attesté, ces auteurs citent les emplois comme prepositional verb "surround, cover, protect, shut, block, oppose, prevent, forbid".
} 
coquillages ou billets de banque... - que le jeune homme offre (hoghog) aux parents de la femme. Néanmoins, on notera que $\mathrm{C}$ et $\mathrm{D}$, même s'ils sont reconstituables, ne prennent jamais la forme d'un syntagme explicite : dans l'emploi normal de ces trois verbes "se fiancer", tout se passe comme si seuls deux participants étaient impliqués, à savoir $\mathrm{A}$ et $\mathrm{B}$.

En somme, dans l'action de bénir comme dans celle de se fiancer, on ne peut pas être sûr si l'on doit poser deux ou quatre participants. Dans ce cas comme dans d'autres, il faut accepter de considérer que l'on a des cas intermédiaires, où un participant n'est ni tout à fait impliqué dans une configuration, ni tout à fait absent. Il ne s'agit pas là d'une défaillance de la théorie, mais d'une prise en compte, dans la perception du monde, des différents degrés d'implication des participants dans le cadre du procès. C'est d'ailleurs cette variabilité sémantique qui permet de passer imperceptiblement, par exemple, du sens $n^{\circ} 1$ [A recouvre $B$ avec un instrument externe C] au sens $n^{\circ} 4$ [A recouvre lui-même B] - cf. Annexe.

Les sujets humains perçoivent et conçoivent des situations en nombre infini, dans lesquelles les éléments du monde se trouvent combinés à loisir. La difficulté que rencontre tout locuteur est de devoir traduire cette infinité, qui est de l'ordre du continu, en un nombre fini de représentations et de signes linguistiques, qui sont de nature discrète $^{14}$. Le propre des structures d'une langue donnée est de proposer certaines catégorisations par défaut, afin de simplifier le travail d'expression du sujet locuteur : verbes traités comme a priori intransitifs vs. transitifs, systèmes de relateurs en nombre réduit, etc. Mais derrière ces catégorisations opérées par chaque langue, il importe au linguiste - à la manière de l'auditeur de l'énoncé - de pouvoir toujours reconstituer la continuité profonde qui unit entre eux ces éléments discrets, à un niveau cognitif que l'on peut appeler perception pré-linguistique du monde.

En ce qui concerne notre goy, cette perspective théorique doit nous permettre de ne pas confondre deux choses :

- la structure syntaxique des énoncés produits, dont les catégories sont discrètes : nombre $n(0 / 1 / 2 \ldots)$ de compléments, présence $\left[\mathrm{n}^{\circ} 1\right]$ ou non [n² à 4] d'un instrumental.

- la valeur référentielle de ces mêmes énoncés, où les gradations et continuums sont monnaie courante : participants plus ou moins impliqués, plus ou moins suggérés par le sémantisme du verbe, plus ou moins autonomes les uns par rapport aux autres [ex. si je me bouche les oreilles avec les mains, a-t-on 1 seul participant, ou 2 ou 3 ou 4 ?]

Précisément, s'il est vrai que nous avons, jusqu'à présent, concentré notre attention sur la signification de goy, et sur les mille facettes de ce kaléidoscope sémantique, il est temps d'observer de plus près la façon dont cette multiplicité de significations se trouve insérée dans le moule étroit de la phrase motlav. Qu'implique donc ce passage, pour ainsi dire, de l'infini référentiel au fini syntaxique ?

\footnotetext{
${ }^{14}$ Nous nous inspirons de la théorie sémantique d'Alain Lemaréchal, autour du pointillisme ou "points mis pour une surface" (1998a: 220, 225). Voir aussi la critique que fait Margetts (1999: 58) des représentations scalaires en typologie.
} 


\section{Syntaxe : rôles sémantiques et valence}

\subsection{Syntaxe de la prédication en motlav}

Avant d'entrer dans le détail des questions de syntaxe relatives au morphème goy, quelques notes grammaticales vont nous permettre de nous situer au sein d'un système.

Le motlav est une langue strictement SVO, dans laquelle le verbe est soit monovalent $(\mathrm{SV})$, soit bivalent (SVO), sans possibilité de verbe trivalent à deux objets directs, du type I wrote the teacher a letter. La fonction S/O est indiquée exclusivement par la place du mot, sans morphème casuel. Outre le COD, le motlav peut faire suivre le verbe de divers compléments, soit directs (Locatifs), soit introduits par une préposition : (Directionnel +) hiy Datif ; bE- Final ; mi Instrumental ; den Ablatif... Cf. (4) supra.

Si la place de Sujet n'appelle pas de remarque particulière, celle de V dans la formule SVO, doit être davantage précisée. Mieux vaut parler ici de Syntagme Prédicatif (SPrd), pour plusieurs raisons :

- d'autres parties du discours que le verbe sont prédicatives en motlav : notamment les noms, adjectifs, locatifs. Il s'agit d'une langue omni-prédicative ;

- le prédicat, qu'il soit verbal ou non, est rarement constitué par un simple mot, mais par un syntagme à trois éléments. Voici ces trois éléments, dans l'ordre de la chaîne :

1] des marques T.A.M. (Temps-Aspect-Mode) pour les prédicats T.A.M. - surtout verbes, adjectifs ;

2] la tête prédicative : verbe, nom, adjectif ; locatif...

3] l'Adjoint du Prédicatif, suivant immédiatement la tête, et modifiant la signification de cette dernière de façon diverse, à la façon d'une épithète.

En résumé, on observe typiquement des énoncés comme celui-ci :

(5) No [mal DĒM veteg ] nē-vēygēl namu-ndō.

je ACP réfléchir (laisser) la-querelle de-nous2

J'ai déjà tiré un trait sur notre querelle (de naguère).

On reconnaît successivement les trois parties principales de la phrase motlav : Sujet ; syntagme Prédicatif ; Compléments (ici le COD). Le SPréd se compose lui-même d'une marque TAM (mal), d'une tête verbale (dēm), et d'un Adjoint du Prédicatif (AdP), le mot veteg.

\subsection{L'Adjoint du Prédicatif : sémantique et valence}

C'est cette position d'AdP qui nous intéresse ici. Celle-ci, propre à la syntaxe du motlav, peut être remplie soit par un verbe (deuxième verbe d'une sérialisation), soit par un adjectif (indiquant la manière de $\mathrm{V}_{1}$ ), soit par un nom (surtout objets incorporés) ; mais on ne peut trouver comme AdP ni syntagme nominal / prépositionnel, ni complément d'aucune sorte. Et l'on notera qu'aucun complément ne peut séparer un AdP du verbe précédent.

D'autre part, certains mots se rencontrent exclusivement dans cette position, et ne sont ni noms, ni adjectifs, ni verbes : c'est le cas du suffixe galsi "(faire $\mathrm{V}_{1}$ ) comme il faut", ou de veteg en (5). C'est enfin le cas - ne le perdons pas de vue - de notre goy, 
dans tous ses emplois : la seule position syntaxique où apparaît ce morphème, est celle d'Adjoint du Prédicatif.

Tous ces AdP ont pour point commun d'ajouter certains sèmes à la tête prédicative : qu'il s'agisse de "(faire $\mathrm{V}_{1}$ ) comme il faut" / "en même temps qu'une autre action $\mathrm{V}_{2}$ " / "par réaction"..., le premier apport de ces AdP est d'ordre sémantique. Cependant, dans certains cas, cet apport sémantique s'accompagne d'une reconfiguration syntaxique regardant la valence de $\mathrm{V}_{1}$. C'est le cas, revenons-y un instant, en (5) : alors que dēm "réfléchir" est lui-même un verbe intransitif dans cet emploi, veteg le transitivise, en donnant au syntagme verbal dans son ensemble une valence double dēm-veteg "réfléchir de façon à volontairement laisser (qqch)" > "oublier (volontairement), pardonner". Ce comportement est le même si $\mathrm{V}_{1}$ est un verbe de mouvement monovalent :

(6) Nēk [ma- VAN veteg ] kemem ba-hap ? tu PFT aller (laisser) nous pour-quoi Pourquoi nous as-tu abandonnés? [tu es parti laissé nous]

Même si veteg n'est pas - ou plus ${ }^{15}$ - un verbe en synchronie, il a du moins le pouvoir de modifier la valence du verbe qu'il accompagne. Son comportement fait penser à celui de certains préverbes latins, dont l'effet est parfois de rendre transitif un verbe intransitif ; Ernout \& Thomas (1953: 21) citent notamment les "verbes composés" ad-ire, ob-ire, ad-loqui, per-currere, tous suivis de l'accusatif, contrairement à leur verbe simple. Les deux auteurs expliquent cette "influence de la composition" par le fait que :

en plus de l'idée de mouvement qui souvent subsiste, la notion évoquée par le composé est celle d'un verbe transitif : par exemple, inire uiam ou suscipere negotium impliquaient l'idée d'un verbe "faire" [nous soulignons, A.F.]

En des termes plus modernes, on dira que l'ajout d'un Adjoint du verbe (préverbe en latin) crée une relation actancielle supplémentaire, en sorte que le procès se trouve en quelque sorte "indexé" sur un nouveau participant, qui se trouve affecté par ce procès.

\subsection{Des morphèmes applicatifs ?}

C'est exactement le cas de veteg en (6) : alors que l'action de partir n'implique qu'un seul participant (celui qui part), l'ajout de cet AdP permet en outre d'indexer cette même action vers un second participant, à savoir la personne (kemem) que l'on quitte en partant. Ce comportement syntaxique de diathèse progressive rappelle assez bien le problème des Applicatifs, ces morphèmes intraverbaux permettant de promouvoir à la place d'objet un participant extérieur (?), qui serait sans cela traité comme un circonstant ${ }^{16}$. Néanmoins, bien que cette comparaison avec les Applicatifs soit séduisante, elle a l'inconvénient de franchir trop vite la distance qui sépare le lexical du grammatical : alors que le propre d'un Applicatif est d'être compatible avec un grand nombre de verbes et de relations différentes, un mot comme veteg continue, malgré tout, à n'être employé que dans un très petit nombre de cas (faibles en extension, larges en compréhension).

\footnotetext{
${ }^{15}$ Une enquête auprès de langues voisines comme le mosina (8 locuteurs, cf. François 1999) donne pour veteg une valeur de verbe transitif "poser, déposer".

${ }^{16} \mathrm{Cf}$. le peul, le nahuatl, le kinyarwanda.
} 
En outre, comparons les trois énoncés suivants, dans lesquels l'AdP a un effet transitivant sur le verbe $\mathrm{V}_{1}$ "aller" :

(7a) Kē [ma- van veteg $]$ na-gasel nono-n. il PFT aller (laisser) le-couteau de-lui Il est parti en oubliant son couteau.

(7b) Kē [ma- van têy ] na-gasel nono-n. il PFT aller tenir le-couteau de-lui Il est parti avec son couteau à la main.

(7c) Kē [ma- van goy ] na-gasel nono-n. il PFT aller ? le-couteau de-lui Il est parti chercher son couteau.

Il est donc difficile de parler d'Applicatif pour veteg, car sa valeur reste largement spécifique. Nous dirons d'ailleurs la même chose pour goy, même si l'élargissement sémantique lié à certains de ses emplois rend bien tentante cette étiquette. Du moins, pouvons-nous désormais inscrire dans ce type de problématique actancielle, les remarques suivantes sur la syntaxe de ce goy.

\subsection{Le jeu des chaises musicales}

Le mécanisme de diathèse progressive que nous venons de montrer avec veteg est simple, et ne semble pas poser de problème. En effet, ce mot vient ajouter un actant supplémentaire à un verbe $\mathrm{V}_{1}$ qui est normalement monovalent, puisqu'il s'agit surtout de verbes de mouvement (aller, partir, courir, sauter...) ; dès lors, la combinaison de $\left\{\mathrm{V}_{\text {aller }}(\mathrm{x})+\operatorname{AdP}_{\text {laisser }}(\mathrm{x}, \mathrm{y})\right\}$ donne naturellement un verbe complexe

$$
\left\{[\mathrm{V}+\mathrm{AdP}]_{\text {quitter }}(\mathrm{x}, \mathrm{y})\right\}
$$

à deux places d'arguments. Ce verbe complexe pourra se co-lexicaliser, sans difficulté, sous la forme d'un verbe transitif comme un autre: ainsi yow-veteg "bondir-laisser" s'est lexicalisé comme verbe bivalent "fuir (qqn, qqch)". L'essentiel, ici, est de constater combien cette première combinaison est simple, puisqu'un prédicat monovalent $f(\mathrm{x})+$ un prédicat bivalent $g(\mathrm{x}, \mathrm{y})$, dans la mesure où ils partagent un de leurs arguments, donneront sans difficulté un nouveau prédicat bivalent.

L'affaire se corse avec un morphème comme goy. D'une part, nous avons vu que ce mot n'ajoute pas un seul nouvel argument, mais deux, voire trois. Or, nous avons vu que le motlav interdit les verbes à plus de deux places d'arguments : on se retrouve donc dans un jeu de chaises musicales, où trois participants doivent s'asseoir sur deux chaises au maximum ${ }^{17}$. Le restant n'a pas d'autre choix que de figurer en dehors du syntagme verbal, et de trouver une nouvelle relation $f$ susceptible de l'accueillir : ce sera généralement le cas avec une préposition (den, mi...).

Ainsi, si l'on reprend nos exemples et nos notations du début, on formulera l'exemple (1') ainsi :

\footnotetext{
${ }^{17}$ Nous avions déjà développé des raisonnements similaires en théorie phonologique (François 1999) : il s'agissait de faire entrer des phonèmes dans des schémas syllabiques contraignants, à nombre de places limité. Ces phénomènes de "chaises musicales" mettent à jour la tension fondamentale, à l'œuvre dans tout le langage, entre un désir de "tout dire" qui tend vers l'infini et, d'autre part, des catégories simples et limitées, en nombre beaucoup plus restreint.
} 


\begin{tabular}{|c|c|c|c|c|c|}
\hline$V_{l}$ & & $A d P$ & & $V_{l}-A d P$ & Préposition \\
\hline $\begin{array}{l}\text { hag } \\
\text { assis }\end{array}$ & $x$ & $\begin{array}{c}\text { goy } \\
\text { s'interposer }\end{array}$ & $=$ & $\begin{array}{l}\text { hag-goy } \\
\text { occulter }\end{array}$ & $\begin{array}{c}+\quad \text { den } \\
\text { éloigné }\end{array}$ \\
\hline A & & A B D & & A B & B D \\
\hline
\end{tabular}

Parmi les diverses relations existant entre les participants (A-B, B-D, A-D, A-B-D), l'énoncé final n'en code que deux, nécessairement binaires - puisque les relateurs ternaires n'existent pas. Il en résulte une réorganisation de toute la valence du groupe, et un décumul des relations actancielles sur plusieurs morphèmes ${ }^{18}$.

Ce n'est pas tout. Nous venons de prendre un exemple simple, dans lequel $\mathrm{V}_{1}$ était lui-même intransitif. Mais que se passe-t-il si ce premier verbe a un complément d'objet ? Ce cas de figure est représenté par les ex. (3')/(4) ci-dessus. On a le schéma suivant :

\begin{tabular}{|c|c|c|c|c|c|c|}
\hline$V_{l}$ & & $A d P$ & & $V_{l}-A d P$ & Prépl & Prép2 \\
\hline $\begin{array}{l}\text { hey } \\
\text { revêtir }\end{array}$ & $x$ & $\begin{array}{c}\text { goy } \\
\text { interposer }\end{array}$ & $=$ & $\begin{array}{l}\text { hey-goy } \\
\text { protéger }\end{array}$ & $\begin{array}{l}+\quad \mathbf{m i} \\
\quad \text { utiliser }\end{array}$ & $\begin{array}{l}\text { (+ den) } \\
\text { (éloigné) }\end{array}$ \\
\hline $\mathrm{AC}$ & & A B C (D) & & A B & A C & (B D) \\
\hline
\end{tabular}

\subsection{La stratégie sérielle}

Outre la préposition, une stratégie légèrement différente employée pour décumuler les relations est de créer une place supplémentaire en coordonnant deux propositions, l'un des deux verbes assumant l'une des relations en jeu :

(8)

$\begin{array}{llll}\text { Nēk } & \text { yēt } & \text { no-yot } & \text {... } \\ \text { tu } & \text { nouer } & \text { les-feuilles:de:sagoutier } \\ \text { Tu attaches } & \text { ensemble les feuilles } & \text { [de sagoutier] }\end{array}$

(8') Na-tmatwoh, lep no-yot tō yēt goy na-mtan. le-tambour prendre (feuilles) alors nouer goy son:œil Le tambour (à membrane), on prend des feuilles et on (les) attache sur / on (en) recouvre son ouverture.

En (8), la relation entre A (celui qui fabrique le tambour à membrane) et $\mathrm{C}$ (les feuilles de sagoutier) est assurée normalement par le verbe transitif yēt "nouer" : on n'a pas de mal à y voir un Agent et un Patient, du moins dans cette première phrase. Mais en (8'), l'adjonction de goy évince ce même $\mathrm{C}$ de la place d'objet de yèt, en réorientant l'ensemble yēt-goy vers un autre participant (orientation secondaire); la place unique d'objet de ce verbe est alors réservée à $\mathrm{B}$, second élément affecté par l'opération, à savoir l'orifice du tambour. En termes fillmoriens, une question fondamentale consisterait à se demander si $\mathrm{B}$ reste le "Bénéficiaire" de l'action yètgoy, et $\mathrm{C}$ son Patient (on aurait alors un cas d'Applicatif ?), ou bien si l'on n'assiste pas tout simplement à la définition de nouvelles actions : au bout du compte, l'action de yèt-goy est envisagée non pas dans ses effets sur $\mathbf{C}$ - qui est donc ravalé au rang d'Instrument - mais dans ses effets sur B - qui se trouve promu non seulement comme Objet en termes syntaxiques, mais également, c'est important, comme Patient en termes sémantiques.

\footnotetext{
${ }^{18}$ Pour cette notion, et le cadre théorique de notre analyse, voir Lemaréchal (1998a, 1998b).
} 
Quoi qu'il en soit, la relation entre $\mathrm{A}$ et $\mathrm{C}$ se trouvant périphérisée hors du procès yēt-goy, celle-ci doit être soit simplement implicitée, soit représentée ailleurs dans le contexte. C'est là le rôle de la première proposition lep no-yot, laquelle rappelle nettement les séries verbales de langues comme le chinois ou le yoruba ${ }^{19}$, et à vrai dire de bien d'autres langues encore. On obtient le schéma :

\begin{tabular}{|c|c|c|c|c|c|c|}
\hline$V_{l}$ & & $A d P$ & & $V_{o}$ & & $V_{l}-A d P$ \\
\hline $\begin{array}{c}\text { yēt } \\
\text { nouer }\end{array}$ & $x$ & $\begin{array}{c}\text { goy } \\
\text { entourer }\end{array}$ & $=$ & $\begin{array}{c}\text { lep } \\
\text { prendre }\end{array}$ & + & $\begin{array}{l}\text { yēt-goy } \\
\text { recouvrir }\end{array}$ \\
\hline A C & & A B C & & A C & & A B \\
\hline
\end{tabular}

On voit donc qu'un suffixe verbal comme goy, du fait de son sémantisme fondamental à quatre participants, est capable d'occasionner de profondes restructurations syntaxiques au sein de la phrase. Allons plus loin : s'il y a un sens à chercher une priorité dans les opérations cognitives, gageons que, pour constituer un énoncé particulier, la sémantique se situe toujours en amont de la syntaxe - laquelle se plie tant bien que mal à un projet énonciatif constitué en dehors d'elle. Bien sûr, cette idée appelle la discussion.

\section{Diathèse et prototype}

\subsection{Une orientation ambiguë}

Cependant, le point le plus original concernant la syntaxe de goy reste à venir. Jusqu'ici, tous nos raisonnements semblaient admettre le même mécanisme: le groupe verbal $[V+$ goy] constitue un verbe transitif, dont le Patient est un objet qui se trouve sémantiquement recouvert/ protégé, c'est-à-dire $B$; tout autre participant est exclu de la position de COD, et en particulier l'ancien Patient de V se trouve éjecté par cette opération. Enfin, un dernier participant introduit par ce même goy, à savoir $D$ (l'agresseur), se retrouve périphérisé, en position de circonstant.

Cette description est exacte pour une bonne partie des emplois de goy, environ la moitié : les effets de sens "entourer / protéger / se fiancer / bénir / aller chercher / pour..." [cf. Annexe, $\mathrm{n}^{\circ} 1$ à 11], sont tous justiciables de cette même analyse syntaxique. Et pourtant, la surprise que nous offrent tous les autres emplois de goy [ ${ }^{\circ} 12$ à 20], c'est de ne plus être orientés - au sens de l'orientation secondaire, vers l'Objet (Lemaréchal 1989) - vers le protégé (B), mais vers l'agresseur (D).

Pis encore : le même verbe complexe $\left[\mathrm{V}_{1}+\right.$ goy] pourra être suivi d'un complément d'objet référant tantôt à $\mathrm{B}$, tantôt à $\mathrm{D}$ :

(9) Kēy mi-tig goy na-tmat den Wilson. ils PFT-debout goy les-masques ABL W. Ils ont protégé les masques sacrés contre Wilson.

\footnotetext{
${ }^{19}$ Cf. l'ex. Mo fi àdè bé igi "I took machete cut tree", de Givón (1984: 179), repris par Lemaréchal (1998a: 207). Cependant, nous réservons le terme de série verbale en motlav à une combinaison $\mathrm{V}_{1}-\mathrm{V}_{2}$ au sein du groupe Prédicatif, et donc sans aucun objet séparant ces deux verbes; l'ex.(8') est simplement une séquence de deux propositions coordonnées.
} 


\section{(9') Kēy mi-tig goy Wilson hag le-tno plēn. ils PFT-debout goy W. Est à-lieu avion Ils ont barré la route à Wilson là-bas, devant l'aéroport.}

Ces deux énoncés relatent le jour où les chefs de Motalava empêchèrent un anthropologue américain d'emporter hors de l'île les masques sacrés qu'il venait d'acheter. En (9), l'objet du verbe complexe tig-goy est B, en sorte qu'on le traduira "protéger", "sauver" ; en (9'), le même verbe est orienté vers D, et il s'agit cette fois non plus d'agir pour quelqu'un, mais contre.

Ce dernier cas de figure, à savoir le verbe [V+goy] orienté vers l'agresseur D, est à la source de multiples effets de sens : empêcher, interdire, tenir à l'écart; mais aussi des valeurs plus abstraitement détrimentales, d'une part, et réactives, d'autre part. On comprend maintenant pourquoi le même morphème goy, dans le Tableau p.3, présentait paradoxalement des valeurs tantôt Bénéfactives [bénir; se couvrir (le visage); surveiller], tantôt Détrimentales ou assimilées [interdire ; retenir qqn; se reposer de] - et ce, avec parfois les mêmes verbes [respectivement vap, tēy, mitiy].

\subsection{Efficacité du prototype}

En ce qui concerne les emplois particuliers de ce goy, que nous ne pourrons développer ici, nous renvoyons encore une fois aux exemples donnés en Annexe. Simplement, dans la lignée de notre démonstration sur la valence et la diathèse liées à ce morphème, nous nous interrogerons sur les critères précis permettant de distinguer le rôle sémantique de B vs. D, dans des couples d'énoncés comme (9)-(9'). En effet, si l'objet de $[\mathrm{V}+\mathrm{goy}]$ était systématiquement $\mathrm{B}$, comme on a pu le croire d'abord, alors ce B serait facilement reconnaissable - selon un raisonnement d'ailleurs circulaire par sa fonction syntaxique d'objet; mais ce n'est pas le cas. Sur quels critères nous basons-nous donc pour classer les emplois abstraits de goy, et décider qu'ils impliquent B vs. D ? Et du point de vue théorique, est-il raisonnable de conserver cette distinction entre deux rôles sémantiques, lors même que leur traitement formel ne semble pas les distinguer particulièrement?

C'est ici qu'intervient à nouveau la notion de prototype. Lorsque nous avons défini le scénario typique de goy [\$2.3], nous avons volontairement éliminé l'analyse componentielle en termes de conditions nécessaires et suffisantes d'apparition de ce mot. Nous avons préféré adopter une approche cognitiviste, dans laquelle les liens unissant toutes les acceptions de goy ne sont pas de l'ordre du vrai / faux, mais du semblable / dissemblable. En particulier, nous avions défini chacun des quatre participants A B C D par un ensemble de traits prototypiques: A serait plutôt <HUMAIN ACTIF>, etc. Or, nous soutenons que le prototype continue d'exercer sa "puissance attractive", pour ainsi dire, même dans les emplois les plus abstraits / dérivés / métaphoriques d'un morphème.

En l'occurrence, un trait qui permet de tracer la frontière entre $\mathrm{B}$ et $\mathrm{D}$, est le fait que B sera toujours du côté de l'inanimé non-agentif - objet à protéger [cf. sens $\mathrm{n}^{\circ} 6$; 7], lieu [ ${ }^{\circ} 3$ ] ; personne soumise $\left[n^{\circ} 7 ; 8\right]$, malade ou fragile [ ${ }^{\circ} 9$ à 11$]$ - tandis que $\mathbf{D}$ se reconnaîtra systématiquement à son caractère typiquement animé / actif. Ce trait ACTIF signifie que $\mathrm{D}$ est toujours celui qui essaye de faire une action $V_{D}$, à laquelle $A$ vient réagir: d'où les valeurs "empêcher", "interdire", mais aussi "en réaction" [n¹6 à 19]. 


\subsection{Illusions d'optique}

L'intérêt de cette approche par le prototype, est qu'elle permet de retracer le cheminement cognitif d'un énoncé comme le suivant :

(10) Ige tā̄an haw goy na-he-k.

les hommes danser goy ma:chanson

Les hommes viennent danser sur ma chanson.

( sur la chanson que l'on chante en mon honneur)

En effet, que faire ici de na-he-k ? C'est l'objet du verbe complexe haw goy "danser le no-yoñyep sur (telle ou telle musique)", mais quel rôle sémantique lui attribuer à l'origine, si l'on admet notre schéma à quatre participants - et que l'on est prêt à jouer le jeu jusqu'au bout? À première vue, il n'y a pas plus de raison de voir en cette chanson un B (un protégé) qu'un D (un agresseur), pas plus d'ailleurs qu'un C (une protection)... On est alors tenté d'abandonner la partie, et de considérer que cet objet vient de nulle part. Au mieux, rapprochant cet emploi du $\mathrm{n}^{\circ} 11$, on fera de goy un Applicatif, susceptible d'ajouter une place d'argument à n'importe quel verbe intransitif, quel que soit le sémantisme des actants.

L'ennui, c'est que cette dernière solution est fausse : goy a certes pour effet de transitiver un grand nombre de verbes monovalents, mais pas tous. Qui plus est, cette analyse fait comme s'il y avait un sens à vouloir "transitiver" un verbe de façon mécanique, indépendamment des sèmes en jeu. En réalité, la clef de l'exemple (10) se trouve dans le sens du nom na-he-k ("chanson en mon honneur"), ou plus exactement dans sa valeur précise en contexte : car "danser sur une chanson", implique nécessairement que cette chanson est en train d'être chantée au moment même de la danse. La danse est donc une forme de réaction de $A$ à une action d'autrui (le chant), et peut donc être rapprochée d'autres exemples $\left[\mathrm{n}^{\circ} 16,17\right]$ où goy acquiert certaines valeurs aspectuelles d'ailleurs tout à fait remarquables : à partir de la valeur primitive $U n$ agent $A$ réagit à une première action (agressive ou non) d'un autre agent $D$, se constituent des mécanismes de repérages inter-procès - du type "Moi j'envoie, et toi tu reçois." / "Toi tu chantes, et moi je danse (en réaction)". Dans ce cas de figure, goy est devenu sinon obligatoire, du moins ordinaire, pour marquer la simultanéité / complémentarité de deux procès $\mathrm{P}_{1}$ et $\mathrm{P}_{2}$. On mesure l'incroyable chemin parcouru, pour atteindre ces significations d'ordre aspectuel, depuis les premières significations de goy, type recouvrir, protéger - pour ne rien dire de l'étymologie *koro "village fortifié" !

En revanche, si c'est bien un "D" qu'il faut reconstituer dans l'exemple (10), une analyse différente est nécessaire pour expliquer les énoncés regroupés sous $\mathrm{n}^{\circ} 11$ : car dans une phrase comme "Il s'est mis en colère pour (défendre) son fils", le fils en question est beaucoup plus proche d'un participant de type B (protégé, inactif, soumis...) que de type D. En sorte que, malgré les apparences, il faudra analyser séparément deux emplois de goy $\left[n^{\circ} 11 \neq 18\right]$ qu'une approche étroitement syntaxique aurait trop tôt assimilés : se prévalant de l'effet "transitivant" de goy dans les deux cas, on eût été tenté de rapprocher les deux types d'exemples, au risque de mettre à mal la prégnance du scénario global que nous avions proposé pour ce mot [§ 2.3]. 


\title{
5. Conclusion : que la syntaxe n'est pas première
}

Dans cette étude de goy en motlav, il est apparu que l'analyse linguistique ne peut pas se contenter de partir des structures pour remonter aux énoncés particuliers, mais doit faire exactement l'inverse : en postulant comme unité fondamentale l'énoncé contextualisé, on cherche à définir précisément les sèmes qui, disséminés dans chaque situation réelle, accompagnent régulièrement telle ou telle marque. Par exemple, malgré ses allures de morphème grammatical, il est impossible de définir goy en termes de valence syntaxique (type Applicatif), ou d'y voir un exemple de "relateur" sans limites précises. En fait, l'apparition de ce morphème dans le discours est liée à des indices contextuels précis - mais prototypiques - qui opèrent bien avant l'insertion dans des structures phrastiques contraignantes : présence d'un danger; désir de protéger un objet prisé; attitudes psychologiques défensives, réaction à une action étrangère \pm offensive; notion d'obstacle, etc.

Ceci nous persuade encore davantage que les relations syntaxiques au sein de l'énoncé ne se situent jamais en amont de la formulation, mais plutôt - pour peu que cette formule ait un sens - en aval. Imaginer qu'un morphème puisse être en luimême un "pur outil grammatical" (par exemple à effet transitivant), et seulement ensuite se gorger de signification contextuelle, nous semble une perspective erronée sur le fonctionnement de la langue, car elle se priverait d'une épaisseur sémantique indispensable à sa compréhension. Certes, goy a des effets transitivants, sur presque tous les verbes qu'il touche; certes, il a également pour effet de réorganiser les structures actancielles au sein de la phrase. Mais, tout bien considéré, ces petits événements syntaxiques ne sont que des épiphénomènes, effets secondaires des véritables réactions chimiques à l'œuvre dans les énoncés : les rencontres de sèmes.

\author{
Alexandre FRANÇOIS \\ LACITO-CNRS / Paris-IV \\ 22, Chemin de la Justice \\ 92290 Châtenay-Malabry \\ <francois@vjf.cnrs.fr>
}

\section{Bibliographie}

BLUST, Robert. 1987. "Lexical reconstruction and semantic reconstruction: the case of Austronesian 'house' words". Diachronica 4, 79-106.

ClARK, Ross. 1985. "Languages of North and Central Vanuatu : groups, chains, clusters and waves". In A. Pawley \& L. Carrington (eds), Austronesian linguistics at the 15th Pacific Science Congress. Pacific Linguistics. Canberra: A.N.U., 199-236.

ERNOUT, Alfred \& THOMAS, François. 1953. Syntaxe latine. Paris: Klincksieck.

FILlmORE, Charles. 1968. "The case for case". In E. Bach \& R. Harms (eds) Universals in Linguistic Theory. New York: Holt, Rinehart \& Winston, 1-90.

FrANCKEL, Jean-Jacques \& LEBAUD, Daniel. 1992. "Lexique et opérations: Le lit de l'arbitraire". In La théorie d'Antoine Culioli: Ouvertures et incidences. L'homme dans la langue. Gap: Ophrys, 89-105.

FRANÇOIS, Alexandre. 1999. "Mouvements et clonages de voyelles en motlav: Entre phonologie et morphologie". Bulletin de la Société de Linguistique XCIV, 1, 437-486. Paris: Klincksieck.

GIVón, Talmy. 1984. Syntax. A functional-typological introduction. Amsterdam / Philadelphia: Benjamins. 
Gross, Gaston. 1999. "Elaboration d'un dictionnaire électronique". Bulletin de la Société de Linguistique XCIV, 113-138. Paris: Klincksieck.

KIMENYI, Alexandre. 1980. A Relational Grammar of Kinyarwanda. Berkeley: University Press of California.

KLEIBER, Georges. 1990. La sémantique du prototype, Catégories et sens lexical. Paris: PUF.

LAKOFF, George. 1987. Women, Fire and Dangerous Things: What Categories reveal about the Mind. Chicago, London: University of Chicago Press.

LEMARÉCHAL, Alain. 1989. Les parties du discours, Syntaxe et sémantique (coll. Linguistique Nouvelle). Paris: PUF.

LEMARÉCHAL, Alain. 1997. "Superposition des marques, zéro et morphologisation". Mémoires de la SLP, $V, 25-61$.

LEMARÉCHAL, Alain. 1998a. Études de morphologie en $f(x, \ldots)$ (coll. Bibliothèque de l'Information Grammaticale). Louvain-Paris: Peeters.

LEMARÉCHAL, Alain. 1998b. "Théories de la transitivité ou théories de la valence: Le problème des applicatifs". In A. Rousseau (ed.) La transitivité. Lille: Presses du Septentrion, 203-218.

MARGETTS, Anna. 1999. Valence and Transitivity in Saliba (coll. MPI Series in Psycholinguistics): Max-Planck Institut.

PAWLEY, Andrew. 1976. Some new Oceanic comparisons (non publié).

PAWLEY, Andrew. 1993. "A language which defies description by ordinary means". In W. Foley (ed.) The role of theory in language description. Trends in Linguistics. Berlin: Mouton de Gruyter, 87-129.

PAWLEY, Andrew. 1996. "Grammarian's lexicon, lexicographer's lexicon: Worlds apart". In J. Svartvik (ed.) Words: Proceedings of an International Symposium. Konferenser. Stockholm: Kungl. Vitters Historie och Antikvitets Akademien, 189-211.

TOURATIER, Christian. 1996. "Sémème, polysémie et théorie du prototype". Bulletin de la Société de Linguistique XCI, 77-96. Paris: Klincksieck.

LEXICAL DERIVATION AND VALENCY-CHANGING STRATEGIES: TAILORING SYNTAX TO SEMANTICS - The description of unknown languages reveals the methodological importance, including for general and typological linguistics, of considering individual contextualised sentences as the fundamental unit for the observation of meaning. This method proves to be powerful in analysing the very meaning and mechanisms associated to an incredibly challenging suffix in Motlav (Oceania). This verbal suffix goy recalls the syntax-andsemantics issues related to preverbs in European languages, to serial verbs or to applicative morphemes found in several parts of the world. Therefore, this study approaches both realms of lexical semantics and of syntactic transitivity.

Our definition of the suffix goy takes the form of a "prototypical scenario", involving four semantic roles A B C D, mutually related by specific mood attitudes. Through metaphoric or metonymic patterns, Motlav derives from this scenario a fair number of semantic values, in accordance with each context. Starting from this first semantic stage, we show the kind of selections and transformations implied when the speaker inserts this scenario into the limited syntactic structures : the necessity of matching semantic roles with grammatical functions triggers several valency-reshaping strategies inside the sentence.

In an attempt to give a model for the cognitive operations involved by linguistic communication, we eventually consider syntax to be a secondary stage in the process of formulation. First of all comes the non-limited, fuzzy representation of a semantic project; then, the syntactic stage provides a set of limited, discrete categories, which just adapt as they can to fit that semantic project. 
NAMYŌS MINO, SO NOK SO DĒMDĒM HIY NA-VAP TO-M̄MOTLAP VITWAG, A SO GOY EN Veg M̄otlap gēn yon̄yoñteg vel qō̄n no-hohole qele so 'Etgoy kē', si so Nok higgoy nēk' si so 'Kemem hoghoggoy na-lqōvēn', si 'Kēy tō-m̄̄ol la-halgoy', $b a$ Nok so salsalgoy n-ēm mino'. Ba nē-vèvēhiy mino so : na-manig ne hohole en na-hap ? Veg na-vap non ige qagqag ne-tegha, na-vap te-le-lam ne-tegha, na-vap nongēn ne-tegha. Ba nok so galeg no-yobem liwo bi-tiy gatgat nongēn en, no te-leh no-hohole en mi na-han hohole nonmem?

Bastō nok dēmdēm lōlhiy na-kastom to-M̄otlap, ba ige te le-Pnō Tamtam en kēy togtog namuy qele ave geh, ba vel qōn kēy akakteg geh. L-etet mino, na-manig ne 'goy' en, ni-tiy kè qele gên : so nēk etetgoy na-hapqiyig vitwag, ba n-et tegha so ni-van me so ni-bel si so ni-ak hethet so ni-akteg en, tō inēk nēk tiggoy lok kē so kè tiple tēytēy van na-ge nan en, a nēk boboel goy en. Ba no mal dèm sas ègēn: tō nok èglal so ba-hap tō gēn vap so 'akakgoy na-lqōvēn', so 'dō hohole galingoy den kē', so Nok ti, kēy ti goy', si so 'Kem so haw goy na-he'... No ne-mlaklak a ni-lwo, veg no mal dēm sas ni-tiy dēmdēm ne gatgat nongēn, tō no ta-yap vēh no-yobem vitwag mey nē-wē. Vēwē kimi.

\section{ANNEXE}

Extrait du (futur) Dictionnaire Motlav-Français : entrée goy.

goy $_{1}[$ [əoj] adp. (toujours suffixé à un verbe V). || Schéma gén. un sujet A recouvre un objet $B$ au moyen d'un objet couvrant $C$, spéc. de telle sorte qu'il empêche, volontairement ou non, une tierce personne $\mathrm{D}$ d'accéder à B. D'où $\mathrm{A}$ interpose $\mathrm{C}$ pour protéger $B$ et/ou pour repousser $D$. Les divers sens de goy résultent en partie des diverses combinaisons entre ces éléments.

[POC *koro 'village fortifié ; ceindre']

1) $[A, B, C]$ couvrir, recouvrir, entourer, ceindre (qqch) avec un instrument enveloppant, la nature de cet instrument étant explicitée dans la phrase. • Na-tmatwoh, lep no-yot tō yēt goy na-mta-n. Le grand tambour, on prend des feuilles de sagoutier et on les attache (yēt) de façon à couvrir (goy) son orifice. - Hey goy ni-qti mi na-hat anen! Protège-toi donc la tête avec ce chapeau! - Nēk wēdē goy no mi ne-vet. [conte] Recouvre-moi de pierres (dans le four, cf. wdē).

2) $[A, B,(C)]$ idem, la nature de l'instrument enveloppant étant suggérée par le verbe $V$. - Kem me-geay goy nē-vētan, kē tale sisgoy soloteg. Nous avons clôturé (geay 'clôture') la terre, de peur qu'elle ne s'effondre de tous côtés. • Kē me-bey goy kē. Elle s'est ceinte d'une jupe (bey).

3) $[A, B,(C)<A]$ recouvrir, boucher: $V$ implique que $A$ couvre $B$ avec une partie de lui-même (hig 'avec le doigt', tēy 'avec la main'...). • Nok tēy goy ne-deln̄e-k. Je me bouche les oreilles (avec les mains). • Nēk mi-sil goy kē! Tu l'as prise dans tes bras!

4) $[A, B]$ recouvrir, envahir (un endroit) : l'objet couvrant est A lui-même. $C f$. prépos. fr. sur. • Nō-wōl me-hey goy na-pnō del. La lune brille sur tout le village. $・ \mathbf{N}-\mathbf{e} \overline{\mathbf{m}}$ ni-yoq goy ne-men è-gēn ! Alors le piège s'effondre sur l'oiseau.

5) $[A, B=C]$ couvrir une chose avec elle-même, d'où : fermer, refermer ; boucler ; joindre, rejoindre. • qul goy no-totgal recoller une photo • gon goy na-mteēm verrouiller la porte • Lok hōw, kē ni-van qal goy mey lok hag. Le côté Ouest va rejoindre le côté Est. • Kē ni-yam goy lok me. Elle règle sa dette envers moi. 
6) $[A, B,(C), D]$ de façon volontaire ou non, agir ou se trouver dans une telle position (verbe $\mathrm{V}$ ) qu'on occulte un objet (B), empêchant du même coup une personne (D) de le voir ou d'y accéder ; $d$ 'où bloquer, occuper, monopoliser ; cacher (qqch), préserver, protéger (qqch) (contre, den) [Obj=B]. • Nēk ma-hag goy na-tnē-k! Tu es assis à ma place [tu es assis-bloques ma place]! • Nēk ma-tañ melmel goy nō-bōk. Avec ta main (tā̄) tu fais de l'ombre (mel) à mon cahier. • Iñglan, na-naw na-hal goy. L'Angleterre est une île [la mer flotte-bloque]. - Ige mayanag mi-hig goy na-tmat so kē nitog leplep. Les chefs ont protégé les masques en empêchant (le touriste) de les emporter.

7) $\left[A, B,{ }^{\prime} C^{\prime},(D)\right]$ protéger (qqch) du contact d'autrui, en employant des paroles consacrées; se réserver (objet, personne, endroit) en le marquant comme sa propriété, s'approprier; rendre sacré ou tabou ; bénir. - No mē-vēg goy so na-kis. Je me le suis réservé pour moi (à manger, kis). • salsal goy n-ēè marquer sa propriété privée sur sa maison [en y accrochant qqch, sal]. • Ige qagqag kēy mē-lēlēp goy Numea. La Nouvelle-Calédonie a été colonisée par les Blancs. • Kem van ak goy na-lqōvēn. Nous allons faire des fiançailles (pour mon fils).

8) $[A, B]$ agir (V) de façon à protéger, obtenir, contrôler (qqch); avoir une position dominante sur; agir sur, agir par rapport à (qqch). • Kem mitiy goy ni-mit. Nous, nous surveillons la viande [en dormant, mtiy]. • Kē no-wotwotmag goy kemem. Il est l'ainé de ("sur") nous tous. • Magra kē ni-lwo goy na-gmel agōh. C'est Magra qui dirige [qui est "grand sur"] ce bar à kava. • No no-bos goy mahgē-k ! C'est moi qui suis mon propre patron [bos], Je suis quand même libre de faire ce que je veux! • Na-Tmat m-aksok so kē ni-wuh Iqet, so kē ni-lwo goy a na-myam kē. Satan cherchait à tuer Dieu, pour devenir le maître du monde.

9) $[A, B]$ agir et spéc. se déplacer pour obtenir (qqch), aller chercher (qqch, qqn). • Na-trak so ni-van goy na-mtewot! Il faut une voiture pour aller chercher un blessé! • Kēy tēvēy goy nō-mōmō. Ils plongent chercher du poisson (ils font de la pêche sous-marine). • Nok vēhge goy ne-gengen na-ga-mem. Je vais me renseigner sur (goy) le repas que nous allons manger.

10) $[A, B]$ agir (V) de façon à prendre soin de (qqn) ; s'occuper de, soigner ; aider ; entretenir. - Nēk haghag goy qōtō Makmak veg kē mo-gom. Viens t'asseoir un moment auprès de Makmak, il est malade. • No me-trō̄, ay! Nēk te-pkasteg goy vēh no ? Je suis bourré, dis donc! Tu peux venir m'aider (à rentrer chez moi)? • Na-mtig non Wotlōlan, kē no-dowdow goy vaga. Wotlôlan entretient toujours très bien ses cocotiers [en désherbant autour, dowdow] ||Cf. etgoy 'faire attention à, prendre soin de'.

11) $\left[A, B^{\prime}\right]$ valeur Bénéfactive : agir en fonction de (qqn, qqch) : cf. fr. "pour, à cause de". Goy se comporte alors comme un morphème de diathèse applicative. • Kē me-qneg goy nēk. Elle est jalouse pour toi, à cause de toi. • No mo-boel goy inti-k (/No mo-boel inti-k). Je suis en colère pour (défendre) mon fils (/Je suis en colère contre mon fils). • Nok dēmdēm goy nēk aē. Cela me rend inquiet pour toi. ||Cf. dēmdēm goy 's'inquiéter'.

12) $[A,(B), D]$ de façon volontaire ou non, agir ou se trouver dans une telle position (V) qu'on gêne une personne (D), en l'empêchant d'agir, ou d'accéder à qqch (B). D'où : s'interposer contre ; retenir, empêcher ; refuser un droit à, interdire à (qqn) $[\mathrm{Obj}=\mathrm{D}]$. • Roland kē ni-tēytēy goy kōyō. Roland les a empêchés (de se battre). • Kēy mi-tig goy kē hag le-tno baklap gapgap en. C'est là-bas, à l'aéroport, qu'ils lui ont barré la route pour l'empêcher de partir. • Nok hig goy kōmyō so kōmyō tele vanvan hep na-ñye mey gēn. [conte] Je vous interdis d'aller plus loin que la pointe de terre là-bas. I| Cf. gatgoy 'interdit'.

13) $[A, D]$ agir secrètement, à l'insu de (qqn) ; tenir à l'écart de ses actions ou de ses paroles ; comploter contre. • Dō hole loñveg goy Edga! Parlons de façon à ne pas être entendus d'Edgar! | | Cf. halgoy 'secret, société secrète'. 
14) $[A, D]$ valeur Détrimentale : agir de façon gênante, importuner (qqn); $C f$. sens "empêcher". • Nitog wowow goy no. Arrête de me déranger! • Nok hel goy no-hohole no-nmoyō. Je vous interromps. • Na-naw t-ōy goy no. L'océan me séparera (de vous). • Nē-bē ma-mah goy kēy. La rivière les a surpris en baissant soudain de niveau (mah 'sécher') - Nē-dēmdēm mino, ni-vit ni-akak goy. Ma pensée est empêchée / obnubilée par les étoiles. - Tateh age het ni-van goy me. Pas de problème! [Aucun ennui qui survienne] ||Cf. siseg goy 'se jouer de'.

15) $\left[A, D^{\prime}\right]$ si $\mathrm{V}$ exprime le temps qui passe, goy indique que ce passage du temps est défavorable à une action envisagée, qu'il est trop tard; $C f$. '(le temps) joue contre (qqn)'. Par ext. passer (pour le temps), en faveur ou en défaveur de (qqn). - Mahē tō-qō̄n goy qiyig dōyō! [la nuit va tomber sur nous] Ça va nous prendre toute la journée (donc ne commençons pas maintenant)! • N-ēte wo ni-van goy, tō kē oy tō en! Les années pourront bien passer, il continuera à le porter autour du cou!

16) $\left[A,(B),(C), D^{\prime}\right]$ faire qqch $(V)$ par réaction à un événement, réagir ; faire une action pour compenser ou annuler les effets néfastes d'une autre action. Ex. après avoir bu le kava, manger (gen) qqch pour en enlever le goût ; le groupe gen goy a alors pour COD soit ce que l'on mange (C), soit le kava (D), soit la bouche elle-même (B). • ne-gengen so gen goy na-ga aē. / so gen goy na-ple. de la nourriture pour manger 'sur' (compenser) le kava./ pour manger 'sur' (soulager) ta bouche. - Kēy ⿳亠丷厂̄kheg goy ne-legleg. Ils se reposent (à la suite) des fêtes de mariages (qui les ont épuisés). • tēngeg goy $\mathbf{n}$-ē⿳亠丷厂女 soutenir une maison qui risque de s'effondrer (à l'aide de contreforts).

17) $\left[A, D^{\prime}\right]$ agir en alternance avec qqn d'autre ; répondre à une première action d'autrui par une seconde action, de façon unique ou répétitive. • Nok ti, kēy ti goy. Je chante d'abord, puis ils chantent en réponse. - Nok yem n-aranis, ba nēk sēkēskēe goy. Moi je grimpe sur l'oranger, et toi tu réceptionnes (les fruits). - Nok vap na-vap t-ā̄ag, ba nēk sese goy n-eh nan. Moi je raconte le conte, toi tu en chantes la chanson. • Nēk tēy goy, ba kē ni-in. Pendant que tu tiens (la tasse), il boit. • So wo kēy ne-mlaklak goy... S'ils en sont contents... | $C f$. vlu goy 'répondre'.

18) spéc. exécuter telle ou telle danse en même temps qu'une certaine musique; danser sur. • Ige tā̄an haw goy na-he-k. Les hommes viennent danser le no-yoñyep sur ma chanson (composée en mon honneur).

19) $\left[A, D^{\prime}\right]$ avoir tel ou tel comportement $(V)$ de façon systématique, en réaction à une action d'autrui, elle-même répétitive (Cf. Rédup.) ; d'où valeur de répétition. - Nēk vavap me, ba na-lē-k qōōnqōñ goy. Bien que tu me le répètes, j'oublie à chaque fois. • Kē ni-balbal goy n-ēlē-n. Il a l'habitude de se couper les cheveux (s.e. pour les empêcher systématiquement de pousser).

20) [A] faire qqch souvent ou récemment (?), en contexte négatif. • Nok et lāmla⿳亠 goy te ni-gita. Cela fait longtemps que je n'ai pas joué/Je ne joue pas souvent de la guitare. 